TRANSACTIONS OF THE

AMERICAN MATHEMATICAL SOCIETY

Volume 196,1974

\title{
SU $(n)$ ACTIONS ON DIFFERENTIABLE MANIFOLDS WITH VANISHING FIRST AND SECOND INTEGRAL PONTRJAGIN CLASSES $\left({ }^{1}\right)$
}

BY

\author{
EDWARD A. GROVE
}

ABSTRACT. In this paper we determine the connected component of the identity of the isotropy subgroups of a given action of $\mathrm{SU}(n)$ on a connected manifold whose first and second integral Pontrjagin classes are zero and whose dimension is less than $n^{2}-8 n \cdot 3-1$.

1. General introduction. Let $S U(n)$ be a differentiable transformation group acting on a connected manifold $M$ with $\operatorname{dim} M \leq n^{2}-8 n / 3-2$, and assume that the first two integral Pontrjagin classes of $M$ are zero. The purpose of this paper is to determine the orbit structure of $M$ under the action of $\mathrm{SU}(n)$.

To be more precise, in Theorem 2.3 we determine those closed connected simple subgroups $H$ of $\mathrm{SU}(n)$ such that the first integral Pontrjagin class of $\mathrm{SU}(n) / H$, $P_{1}(\mathrm{SU}(n) / H ; Z)$, is zero; in Theorem 2.5 we determine those closed connected simple subgroups $H$ of $\mathrm{SU}(n)$ such that $P_{1}(\mathrm{SU}(n) / H ; Z)=0=P_{2}(\mathrm{SU}(n) / H ; Z)$; in Theorem 2.9 we determine the connected component of the identity of a regular isotropy subgroup of the given above actions of $\mathrm{SU}(n)$ on M. In Theorem 3.1 we then show how the connected component of the identity of all isotropy subgroups of $\mathrm{SU}(n)$ is completely determined by the connected component of the identity of the principal isotropy subgroup, and we give a list of such possible subgroups.

Wu-Chung Hsiang and Wu-Yi Hsiang considered this problem in [4]. One of the key steps in their approach was the ir knowledge of large subgroups of $\mathrm{SU}(n)$. This forced them to put a severe restriction on M. Our approach is more general in nature, and allows us a much less restrictive dimension requirement.

The author wishes to thank his thesis advisor Wu-Yi Hsiang for the encouragement and help he received while writing this paper. Some of these results were included in the author's Ph.D. thesis at Brown University.

In this section we recall several well-known facts. Let $G$ be a compact con-

Received by the editors June 23, 1970 and, in revised form, July 6, 1973.

AMS (MOS) subject classifications (1970). Primary 57E15; Secondary 57E25.

Key words and phrases. Compact transformation groups, inte gral Pontrjagin classes, isotropy subgroups.

(1) Research supported in part by National Science Foundation Grant GP-6371. 
nected Lie group acting differentiably on a manifold $M$. For each $x \in M$, let $G_{x}=$ $\{g \in G: g \cdot x=x\}$ and let $G(x)=\{g \cdot x: g \in G\}$. Then if $f: G / G_{x} \approx G(x) \subseteq M$ is the natural map,

$$
f r(M)=\tau\left(G / G_{x}\right) \oplus \nu\left(G / G_{x}\right)=a_{\xi_{1}}\left(\iota_{G / G_{x}}\right) \oplus a_{\xi_{1}}\left(\phi_{x}\right)
$$

where $\xi_{1}$ is the principal bundle $\left(G_{x} \rightarrow G \rightarrow G / G_{x}\right),{ }^{l} G / G_{x}$ is the isotropy representation of $G_{x}$ in $G$, and $\phi_{x}$ is the slice representation of $G_{x}$ at $x_{0}$. Suppose now that $x \in M$ is a regular element. Then $\phi_{x}$ is a trivial representation [5], and so. $f^{\prime} r(M)=a_{\xi_{1}}\left(\iota G / G_{x}\right) \oplus \theta$ where $\theta$ is a trivial vector bundle. The following result is trivial to prove but is frequently useful.

Proposition 1.1. Let $\eta=(H \rightarrow E \rightarrow B)$ and $\eta^{\prime}=\left(G \rightarrow E^{\prime} \rightarrow B^{\prime}\right)$ be principal bundles, and let

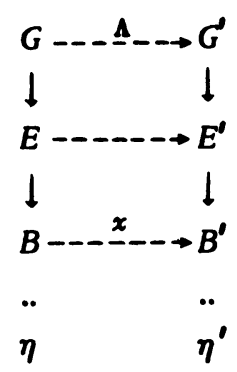

be a bomomorphism of principal bundles. Then

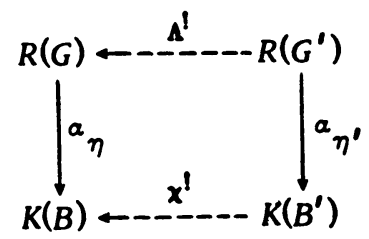

is a commutative diagram.

So if $F: G / G_{x}^{0} \rightarrow G / G_{x}$ is the natural map and $\xi_{2}$ is the principal bundle $\left(G_{x}^{0} \rightarrow G \rightarrow G / G_{x}^{0}\right)$, we have

$$
F^{!} f^{1} r(M)=a_{\xi_{2}}\left(l G / G_{x}^{0}\right) \oplus \theta=r\left(G / G_{x}^{0}\right) \oplus \theta
$$

and so $F^{*}\left(f^{*}\left(P_{j}(M ; Z)\right)\right)=P_{j}\left(G / G_{x}^{0} ; Z\right)$ (where $P_{j}(N ; Z)$ is the $j$ th integral Pontrjagin class of $N$ ).

So to study $G$ actions on a manifold $M$ satisfying $P_{1}(M ; Z)=0=P_{2}(M ; Z)$, we must first find all those closed connected subgroups $H$ of $G$ such that $P_{1}(G / H ; Z)=0=P_{2}(G / H ; Z)$. 
2. Subgroups of $\mathrm{SU}(n)$ with $P_{1}(\mathrm{SU}(n) / H ; Z)=0=P_{2}(\mathrm{SU}(n) / H ; Z)$. Let $G$ be a compact Lie group, $H$ a closed connected subgroup, and let $T$ be a maximal torus in $H$. Then the natural map $\pi: G / T \rightarrow G / H$ is a fibre map with fibre $H / T$, and hence the kernel of the map $\pi^{*}: H^{*}(G / H ; Z) \rightarrow H^{*}(G / T ; Z)$ consists only of torsion elements. Let $\xi$ be the principal bundle $(H \rightarrow G \rightarrow G / H)$ and $\xi^{\prime}$ the principal bundle $(T \rightarrow G \rightarrow G / T)$. Then if $\phi$ is a real representation of $H$, $\pi^{1}\left(a_{\xi}(\phi)\right)=a_{\xi^{\prime}}(\phi \mid T)$ and hence $\pi^{*}\left(P_{j}\left(a_{\xi}(\phi) ; Z\right)\right)=P_{j}\left(a_{\xi^{\prime}}(\phi \mid T) ; Z\right)$. Let $\eta(G)=$ $\left(G \rightarrow E_{G} \rightarrow B_{G}\right)$ be a classifying bundle for $G$. Then $\eta(T)=\left(T \rightarrow E_{G} \rightarrow B_{T}\right)$ is a classifying bundle for $T$. Letting $i: G / T \rightarrow B_{T}$ be the classifying map of $\xi^{\prime}$, we have that $\pi^{1}\left(\alpha_{\xi}(\phi)\right)=i^{1}\left(\alpha_{\eta(T)}(\phi \mid T)\right)$, and hence from [2] that

$$
\begin{aligned}
& \pi^{*}\left(P_{j}\left(\alpha_{\xi}(\phi) ; Z\right)\right)=i^{*}\left(P_{j}\left(\alpha_{\eta(T)}(\phi \mid T) ; Z\right)\right)
\end{aligned}
$$

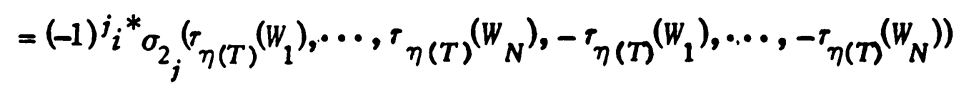

where $\sigma_{2 j}$ is the $2 j$ th elementary symmetric function, ${ }^{\tau} \eta(T)$ is the transgression in the Serre spectral sequence $E(\eta(T))$ associated with $\eta(T)$, and $\left\{W_{1}, \ldots, W_{N}\right\}$ are the positive weight vectors of $\phi$.

Recall now that $r(G / H)=a_{\xi}\left(\iota_{G / H}\right)=a_{\xi}\left(A d_{G} \mid H-\Lambda d_{H}\right)$. By Proposition 1.1, $a_{\xi}\left(\operatorname{Ad}_{G} \mid H\right)$ is a trivial bundle, and hence $r(G / H) \oplus \alpha_{\xi}\left(\operatorname{Ad}_{H}\right)=\theta$ where $\theta$ is a trivial vector bundle.

Corollary 2.1 [2]. Let $T$ be a torus in $G$. Then $G / T$ is stably parallelizable.

From $\pi^{l}(r(G / H))=i^{l} a_{\eta(T)}\left(\iota_{G / H} \mid T\right)$ and $\pi^{!}\left(a_{\xi}\left(\operatorname{Ad}_{H}\right)\right)=i^{l} a_{\eta(T)}\left(\operatorname{Ad}_{H} \mid T\right)$, we have the following corollary.

Corollary 2.2. $\Sigma_{\alpha+\beta=j} \pi^{*}\left(P_{\alpha}(G / H ; Z)\right) \cdot \pi^{*}\left(P_{\beta}\left(\alpha_{\xi}\left(\operatorname{Ad}_{H}\right): Z\right)\right)=0$.

As $E_{G}$ is contractible, $E_{G} \times_{T} G \rightarrow G / T$ is a homotopy equivalence, and hence we may take $\gamma=\left(G \rightarrow G / T \stackrel{i}{\longrightarrow} B_{T}\right)$ to be a principal $G$ bundle. Thus we see that $i^{*}: H^{*}\left(B_{T} ; Z\right) \rightarrow H^{*}(G / T ; Z)$ is just the edge homomorphism

$$
H^{*}\left(B_{T} ; Z\right)=E_{2}^{*, 0}(\gamma) \rightarrow E_{\infty}^{*, 0}(\gamma) \subset H^{*}(G / T ; Z),
$$

and hence $\operatorname{ker} i^{*}=\left\langle\operatorname{im} d(\gamma)^{+}\right\rangle$, the ideal in $H^{*}\left(B_{T} ; Z\right)$ generated by the elements of positive degree in the image of the differential of $E(\gamma)$.

Suppose that $U$ is a closed connected normal subgroup of $H$. Then by using Proposition 1.1 together $w$ ith a standard argument, it follows that $P_{j}(G / H ; Z)=0$ implies that $P_{j}(G / U ; Z)=0$.

So to determine those closed connected subgroups $H$ of $G$ with $P_{1}(G / H ; Z)$ $=0=P_{2}(G / H ; Z)$, we need only determine those closed simple subgroups $U$ of $G$ with $P_{1}(G / U ; Z)=0=P_{2}(G / U ; Z)$ and then, by using the classification theorem 
of compact Lie groups, determine whatever restrictions there are on "piecing" the various simple (we shall see later that if $P_{1}(S U(n) / H ; Z)=0$, then $H$ is either semisimple or a torus) groups together to form an acceptable $H$.

We now let $G=\operatorname{SU}(n)$ and let $\lambda: H \rightarrow \mathrm{SU}(n)$ be the embedding of $H$ as a closed subgroup, i.e. $\lambda$ is an injective homomorphism of the compact connected Lie group $H$ into $\mathrm{SU}(n)$. By examining the spectral sequence $E(\gamma)$ of $\gamma$, we see that in low dimensions at least, $\operatorname{ker} i^{*}=\left\langle\left(\operatorname{im} \tau_{\gamma}\right)^{+}\right\rangle$where $\tau_{\gamma}$ is the transgression of $\gamma_{0}$. Thus we have (at least in low dimensions)

$$
\operatorname{ker} i^{*}=\left\langle S^{+}\left(r_{\eta(T)}\left(\mu_{1}\right), \ldots, \tau_{\eta(T)}\left(\mu_{n}\right)\right)\right\rangle
$$

where $\left\{\mu_{1}, \ldots, \mu_{n}\right\}$ is the set of weight vectors of $\lambda$, and $S^{+}\left(x_{1}, \ldots, x_{n}\right)$ is the ring of symmetric polynomials with zero constant term.

We now apply the above to the representation $\phi=A_{H_{H}}$. Note that as $\lambda: H \rightarrow$ $\mathrm{SU}(n), \mu_{n}=-\left(\mu_{1}+\cdots+\mu_{n-1}\right)$, and hence

$$
2 \sigma_{2}\left(\tau_{\eta(T)}\left(\mu_{1}\right), \ldots, r_{\eta(T)}\left(\mu_{n}\right)\right)=-\sum_{i=1}^{n}\left(\tau_{\eta(T)}\left(\mu_{i}\right)\right)^{2} .
$$

Recall that, by Corollary 2.2,

$$
\begin{aligned}
\pi^{*}\left(P_{1}(\mathrm{SU}(n) / H ; Z)\right)=-\pi^{*} P_{1}\left(\alpha_{\xi}\left(\mathrm{Ad}_{H}\right) ; Z\right) \\
\quad=i^{*}\left(\sigma_{2}\left(\tau_{\eta(T)}\left(W_{1}\right), \ldots, \tau_{\eta(T)}\left(W_{N}\right),-\tau_{\eta(T)}\left(W_{1}\right), \ldots,-\tau_{\eta(T)}\left(W_{N}\right)\right)\right) \\
\quad=-i^{*} \sum_{j=1}^{N}\left(\tau_{\eta(T)}\left(W_{j}\right)\right)^{Z} .
\end{aligned}
$$

So $\pi^{*}\left(P_{1}(\mathrm{SU}(n) / H ; Z)\right)=0$ if and only if for some integer $K$,

$$
K \sum_{j=1}^{n}\left(\tau_{\eta(T)}\left(\mu_{j}\right)\right)^{2}=2 \sum_{j=1}^{N}\left(\tau_{\eta(T)}\left(W_{j}\right)\right)^{2}
$$

So if $U$ is a closed, simple, normal subgroup of $H$, it follows that $\pi^{*}\left(P_{1}(\operatorname{SU}(n) / U ; Z)\right)$ $=0$ if and only if there exists an integer $K$ such that

$$
K \sum_{j=1}^{n}\left|\mu_{j}\right|^{2}=2 \sum_{j=1}^{N}\left|W_{j}\right|^{2}
$$

where || is the Cartan-Killing norm.

We are now ready to determine those closed simple subgroups $G$ of $S U(n)$ for which $P_{1}(\operatorname{SU}(n) / G ; Z)=0$. First note that if $\lambda: G \rightarrow \operatorname{SU}(n)$, then $\operatorname{SU}(n) / \lambda[G]$ is. diffeomorphic to $\operatorname{SU}(n) / \lambda^{*}[G]$. Hence in the following theorem if $\lambda: G \rightarrow \operatorname{SU}(n)$ is such that $P_{1}(\mathrm{SU}(n) / \lambda[G] ; Z)^{\circ}=0$, then we shall either include $(G, \lambda)$ or $\left(G, \lambda^{*}\right)$ in the list, but not both. 
Theorem 2.3. Suppose $\lambda: G \rightarrow \mathrm{SU}(n)$ is an almost faitbful embedding, wbere $G$ is a simply connected, compact, simple Lie group sucb that $P_{1}(\operatorname{SU}(n) / \lambda[G] ; Z)$ $=0$. Then modulo trivial representations, $(G, \lambda)$ appears on the following list:

G

SU(4)

SU(5)

$\mathrm{SU}(6)$

SU(7)

SU(8)

$\operatorname{SU}(k), k \geq 2$

$\mathrm{SU}(k), k \geq 2$

$\mathrm{SU}(k), k \geq 2$

$\mathrm{SU}(k), k \geq 4$

$\mathrm{SU}(k), k \geq 4$

$S_{p}(3)$

$\mathrm{S}_{\mathrm{p}}(k), k \geq 3$

$S_{\mathrm{p}}(k), k \geq 3$

$\mathrm{S}_{\mathrm{p}}(k), k \geq 3$

$S_{\text {PIN }}(5)$

$S_{\text {PIN }}(7)$

$\mathrm{S}_{\mathrm{PIN}}$ (9)

$S_{\text {PIN }}(11)$ $\lambda$

$$
\begin{array}{ll}
b \Lambda^{2} \mu_{4} & b=1,2,4 \\
a \mu_{4} \oplus 3 \Lambda^{2} \mu_{4} \oplus d \mu_{4}^{*} & a+d=2
\end{array}
$$$$
\mu_{s} \oplus b \Lambda^{2} \mu_{5} \oplus c \Lambda^{2} \mu_{5}^{*}
$$$$
b+c=3
$$$$
b \Lambda^{2} \mu_{6} \oplus c \Lambda^{2} \mu_{6}^{*}
$$$$
b+c=1,3
$$$$
b \Lambda^{3} \mu_{6}
$$$$
b=1,2
$$$$
a \mu_{6} \oplus b \Lambda^{2} \mu_{6} \oplus \Lambda^{3} \mu_{6} \oplus c \Lambda^{2} \mu_{6}^{*} \oplus d \mu_{6}^{*}
$$$$
a^{0}+d+4(b+c)=6
$$$$
a \mu_{7} \oplus b \Lambda^{2} \mu_{7} \oplus c \Lambda^{2} \mu_{7}^{*} \oplus d \mu_{7}^{*}
$$$$
a+d=4, b+c=1
$$$$
\mu_{8} \oplus b \Lambda^{3} \mu_{8} \oplus c \Lambda^{3} \mu_{8}^{*}
$$$$
b+c=1
$$

Ad $_{\text {SU(k) }}$

$$
a \mu_{k} \oplus d \mu_{k}^{*}
$$$$
a+d \mid 2 k
$$

$a \mu_{k} \oplus b S^{2} \mu_{k} \oplus c S^{2} \mu_{k}^{*} \oplus d \mu_{k}^{*}$

$a+d=k-2, b+c=1$

$a \mu_{k} \oplus b \Lambda^{2} \mu_{k} \oplus c \Lambda^{2} \mu_{k}^{*} \oplus d \mu_{k}^{*}$

$a+d=2+k, b+c=1$

$a+d=4, b+c=2$

$a+d=2, b+c=1$

$\Lambda^{2} \mu_{k} \oplus S^{2} \mu_{k}^{*}$

$a \Lambda^{2} \nu_{6} \oplus b\left(\Lambda^{3} \nu_{6}-\nu_{6}\right)$

$a+b=1,2$

$4 \nu_{6} \oplus\left(\Lambda^{3} \nu_{6}-\nu_{6}\right)$

$a \nu_{2 k}$

$a \mid 2(k+1)$

$4 \nu_{2 k} \oplus \Lambda^{2} \nu_{2 k}$

$\operatorname{Ad}_{\mathrm{S}_{\mathrm{p}}(k)}$

$a \rho_{s} \oplus b \Delta_{s}$

$2 a+b \mid 6$

$a \rho_{7} \oplus b \Delta_{7}$

$a+b \mid 5$

$a \rho_{9} \oplus b \Delta_{9}$

$a+2 b \mid 7$

$a \rho_{11} \oplus b \Delta_{11}$ 


$$
\begin{array}{lll}
\mathrm{S}_{\mathrm{PIN}}(13) & a \rho_{13} \oplus b \Delta_{13} & a+8 b \mid 11 \\
\mathrm{~S}_{\mathrm{PIN}}(2 k+1), k \geq 2 & a \rho_{2 k+1} & a \mid(2 k-1) \\
\mathrm{S}_{\mathrm{PIN}}(2 k+1), k \geq 2 & \Delta \mathrm{d}_{\mathrm{S}_{\mathrm{PIN}}(2 k+1)} & \\
\mathrm{S}_{\mathrm{PIN}}(8) & a \rho_{8} \oplus b \Delta_{8}^{+} \oplus c \Delta_{8}^{-} & a+b+c \mid 6 \\
\mathrm{~S}_{\mathrm{PIN}}(10) & a \rho_{10} \oplus b \Delta_{10}^{+} \oplus c \Delta_{10}^{-} & a+2(b+c) \mid 8 \\
\mathrm{~S}_{\mathrm{PIN}}(12) & a \rho_{12} \oplus b \Delta_{12}^{+} \oplus c \Delta_{12}^{-} & a+4(b+c) \mid 10 \\
\mathrm{~S}_{\mathrm{PIN}}(14) & a \rho_{14} \oplus b \Delta_{14}^{+} \oplus c \Delta_{14}^{-} & a+8(b+c) \mid 12 \\
\mathrm{~S}_{\mathrm{PIN}}(2 k), k \geq 4 & a \rho_{2 k} & a \mid 2(k-1) \\
\mathrm{S}_{\mathrm{PIN}}(2 k), k \geq 4 & \Lambda \mathrm{d}_{\mathrm{S}_{\mathrm{PIN}}}(2 k) & \\
G_{2} & \phi_{2}=\Lambda \mathrm{d}_{G_{2}}, \phi_{1}, 2 \phi_{1}, 4 \phi_{1} & \\
F_{4} & \phi_{4}=A \mathrm{~d}_{F_{4}}, \phi_{1}, 3 \phi_{1} & \\
E_{6} & a \phi_{1} \oplus b \phi_{5} & a+b=1,2,4 \\
& \Lambda \mathrm{d}_{E_{6}}=\phi_{6} & \\
E_{7} & \phi_{1}, 3 \phi_{1}, \Lambda \mathrm{d}_{E_{7}}=\phi_{6} & \\
E_{8} & A \mathrm{~d}_{E_{8}} &
\end{array}
$$

Remark. Note that if $\bar{G}$ is a compact simple Lie group and $\lambda: \bar{G} \rightarrow G_{1}$ is any nontrivial homomorphism of Lie groups, then $\operatorname{Ker} \lambda$ is a finite central subgroup. So any embedding of $\bar{G}$ lifts to an almost faithful embedding of $\bar{G}$ 's universal covering group $\tilde{\lambda}: \tilde{G} \rightarrow G_{1}$. If $G_{1}=\operatorname{SU}(n)$ and $P_{1}(\operatorname{SU}(n) / \bar{G})=0$, then $(\tilde{G}, \tilde{\lambda})$ will be on the above list.

Proof of Theorem 2.3. Suppose $G=\operatorname{SU}(k), 2 \leq k \leq n$. If $\lambda: \operatorname{SU}(k) \rightarrow \operatorname{SU}(n)$ and $\Sigma(\lambda)=\left\{\left(j, \mu_{j}\right): 1 \leq j \leq n\right\}$ is the set of weight vectors of $\lambda$, let $n(\lambda)=\sum_{j=1}^{n}\left|\mu_{j}\right|^{2}$. For $1 \leq p, q \leq k$, let $e_{q}^{p}$ be the $k \times k$ matrix $e_{q}^{p}=\left[\delta_{p}^{i} \delta_{q}^{j}\right]$. Let $\lambda_{j}=i\left(e_{j}^{j}-k^{-1} \sum_{t=1}^{k} e_{t}^{t}\right)$

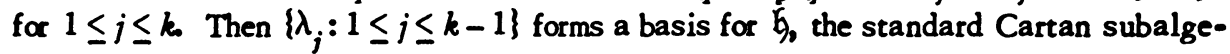
bra of $\mathrm{SU}(k)$. We may take our invariant, nondegenerate inner-product for the real Lie algebra of $\operatorname{SU}(k)$ so that $\left(\lambda_{i}, \lambda_{i}\right)=(k-1) / k$ and $\left(\lambda_{i}, \lambda_{j}\right)=-1 / k$ if $i \neq j$. Note that

$$
n\left(\Lambda \mathrm{d}_{\mathrm{SU}(k)}\right)=2 \sum_{1 \leq i<j \leq k}\left|\lambda_{i}-\lambda_{j}\right|^{2}=2 \sum_{1 \leq i<j \leq k} 2=2 k(k-1) .
$$

So as we have shown, $P_{1}(\operatorname{SU}(n) / \lambda[S U(k)])=0$ implies $2 k(k-1)$ is an integral multiple of $n(\lambda)$.

We let $\pi=\left\{\lambda_{j}-\lambda_{j+1}: 1 \leq j \leq k-1\right\}$ be our system of simple roots. If $\phi$ : 
$\mathrm{SU}(k) \rightarrow \mathrm{SU}(n)$ is irreducible, let $\Lambda_{\phi}$ be the highest weight of $\phi$, and let

$$
q_{j}=\frac{2\left(\Lambda_{\phi}, \lambda_{j}-\lambda_{j+1}\right)}{\left(\lambda_{j}-\lambda_{j+1}, \lambda_{j}-\lambda_{j+1}\right)}=\left(\Lambda_{\phi}, \lambda_{j}-\lambda_{j+1}\right)
$$

Then recall $q_{j}$ is a nonnegative integer.

For each $1 \leq i \leq k-1$, let $\phi_{i}$ be the unique irreducible representation satisfying

$$
\frac{2\left(\Lambda_{\phi_{i}}, \lambda_{j}-\lambda_{j+1}\right)}{\left(\lambda_{j}-\lambda_{j+1}, \lambda_{j}-\lambda_{j+1}\right)}=\delta_{j}^{i}, \text { i.e. } \phi_{i} \leftrightarrow \circ \circ \ldots \circ \text { 1 } \ldots \ldots \circ \infty
$$

Recall that $\phi_{1}=\mu_{k}$ and $\phi_{j}=\Lambda_{\mu_{k}}^{j}, 1 \leq j \leq k$. So

Hence

$$
\sum\left(\phi_{j}\right)=\left\{\lambda_{i_{1}}+\cdots+\lambda_{i_{j}}: 1 \leq i_{1}<i_{2}<\cdots<i_{j} \leq k\right\} \text {. }
$$

$$
\begin{aligned}
n\left(\phi_{j}\right) & =\sum_{1 \leq i_{1}<\cdots<i_{j} \leq k}\left|\lambda_{i_{1}}+\cdots+\lambda_{i_{j}}\right|^{2}=\sum_{1 \leq i_{1}<\cdots<i_{j} \leq k} j\left(\frac{k-j}{k}\right) \\
& =\frac{j}{k}(k-j) \frac{k(k-1) \cdots(k-j+1)}{1 \cdot 2 \cdots j} \\
& =\frac{(k-1)(k-2) \cdots(k-j+1)}{2 \cdot 3 \cdots j} j(k-j) \text { if } j>1 \\
& =\frac{(k-1)(k-2) \cdots(k-j+2)}{2 \cdot 3 \cdots(j-1)}(k-j+1)(k-j) \text { if } j>2 .
\end{aligned}
$$

Note that $n\left(\phi_{j+1}\right)=n\left(\phi_{j}\right) \cdot(k-j-1) / j$. So if $1 \leq j \leq(k-1) / 2$, then $n\left(\phi_{j+1}\right) \geq$ $n\left(\phi_{j}\right)$. Also, $1 \leq j \leq(k-1) / 2$ implies $\Sigma\left(\phi_{j}\right)=-\bar{\Sigma}\left(\phi_{k-j}\right)$ (i.e. $\left.\phi_{j}=\phi_{k-j}^{*}\right)^{j+1}$ which implies $n\left(\phi_{j}\right)=n\left(\phi_{k-j}\right)$. We have

$$
\begin{gathered}
n\left(\phi_{1}\right)=k-1, \quad n\left(\phi_{2}\right)=(k-1)(k-2) \\
n\left(\phi_{3}\right)=1 / 2(k-1)(k-2)(k-3)>2 k(k-1) \quad \text { if } k>9 .
\end{gathered}
$$

So if $4 \leq j \leq[(k+1) / 2]$ and $n\left(\phi_{j}\right) \leq 2 k(k-1)$, it follows that $k \leq 8$, and hence $k=8, j=4$. By a direct check, this is impossible. So if $1 \leq i \leq[(k+1) / 2]$ and $n\left(\phi_{j}\right) \leq 2 k(k-1)$, we have $j=1, j=2$, or $j=3$ and $6 \leq k \leq 8$.

Lemma 2.4. Suppose $\phi: \mathrm{SU}(k) \rightarrow \operatorname{SU}(n)$ is an irreducible map witb $n(\phi) \leq$ $2 k(k-1)$. Then up to conjugation, $\phi$ appears on the following list: 


$$
\begin{aligned}
\Lambda^{3} \mu_{k}, & 6 \leq k \leq 8, \\
\mu_{k}, s^{2} \mu_{k}, \Lambda^{2} \mu_{k}, \text { Ad }_{\mathrm{SU}(k),} & k \geq 2 .
\end{aligned}
$$

Proof. Let

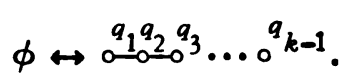

Suppose that there exist at least three distinct indices $a, b, c$ so that $q_{a}, q_{b}$, $q_{c}$ all are different from zero. If $\phi_{1}, \phi_{2}$ are two different irreducible representations, we say $\phi_{1} \geq \phi_{2}$ if $q_{i}\left(\phi_{1}\right) \geq q_{i}\left(\phi_{2}\right)$ for $i=1,2, \ldots, k-1$. It follows easily that $\phi_{1} \geq \phi_{2}$ implies $\left(\Lambda_{\phi_{1}}, \Lambda_{\phi_{1}}\right) \geq\left(\Lambda_{\phi_{2}}, \Lambda_{\phi_{2}}\right)$.

Using this fact, one easily shows $\left(\Lambda_{\phi}, \Lambda_{\phi}\right)>1$.

Since $q_{a}, q_{b}, q_{c}$ are all different from zero, it follows easily by applying the Weyl group that $\Sigma(\phi)$ contains at least $(k-1)(k-2)(k-3)$ distinct vectors $\Lambda$ all with $(\Lambda, \Lambda)=\left(\Lambda_{\phi}, \Lambda_{\phi}\right)$. So $n(\phi) \geq(k-1)(k-2)(k-3)$. So if $k>6, n(\phi)>$ $2 k(k-1)$.

Note $4 \leq k$ as there exist $q_{a}, q_{b}, q_{c} \neq 0$. If $4 \leq k \leq 6$, one shows by direct calculation that $\left(\Lambda_{\phi}, \Lambda_{\phi}\right) \geq 5$ and hence

$$
n(\phi) \geq 5(k-1)(k-2)(k-3)>2 k(k-1) \text { if } 4 \leq k \leq 6 \text {. }
$$

Suppose next that there exist exactly 2 indices $a, b$ with $q_{a}, q_{b} \neq 0$. Suppose $k>9$. Further suppose that $3 \leq a \leq k-3$. Then $\phi \geq \Lambda^{a} \mu_{k}=\phi_{a}$. So $\left(\Lambda_{\phi}, \Lambda_{\phi}\right) \geq\left(\Lambda_{\phi_{a}}, \Lambda_{\phi_{a}}\right)$. Also, by applying the Weyl group, it is clear that we introduce at least as many new vectors $\Lambda$ into $\Sigma(\phi)$ with $(\Lambda, \Lambda)=\left(\Lambda_{\phi}, \Lambda_{\phi}\right)$, as we do vectors $\Lambda^{1}$ into $\Sigma\left(\Lambda^{a} \mu_{k}\right)$ with $\left(\Lambda^{1}, \Lambda^{1}\right)=\left(\Lambda_{\phi_{a}}, \Lambda_{\phi_{a}}\right)$, and so $n(\phi) \geq$ $n\left(\Lambda^{a} \mu_{k}\right)>2 k(k-1)($ as $3<a<k-3)$.

It follows that we may assume (up to conjugation)

(1) $a=1, b=2$,

(2) $1 \leq a \leq 2, k-2 \leq b \leq k-1$,

(3) $1 \leq a \leq 2, b=3,6 \leq k \leq 8$,

(4) $1 \leq a \leq 3, k-3 \leq b \leq k-1,7 \leq k \leq 8$.

By direct computation, we now easily arrive at the result.

With this lemma, the results with $G=\mathrm{SU}(k)$ follow easily. One obtains the results for $G=B_{k}, C_{k}, D_{k}, G_{2}, F_{4}, E_{6}, E_{7}, E_{8}$ similarly.

Theorem 2.5. Suppose $\lambda: G \rightarrow \mathrm{SU}(n)$ is a nontrivial representation, where $G$ is a simply connected, compact, simple Lie group. Suppose $P_{1}(\operatorname{SU}(n) / \lambda[G] ; Z)=0$ $=P_{2}(\mathrm{SU}(n) / \lambda[G] ; Z)$. Then $(G, \lambda)$ (modulo an arbitrary trivial representation) ap. pears on the following list: 
G

$\lambda$

$\begin{array}{lll}\mathrm{SU}(2) & 4 \mu_{2} & \\ \mathrm{SU}(3) & \mu_{3} \oplus b S^{2} \mu_{3} \oplus c S^{2} \mu_{3}^{*} & b+c=1 \\ \mathrm{SU}(4) & \Lambda^{2} \mu_{4} & \\ & 2 \Lambda^{2} \mu_{4} & \\ & \Lambda^{2} \mu_{4} \oplus S^{2} \mu_{4}^{*} & \\ \mathrm{SU}(6) & \Lambda^{2} \mu_{6} & \\ \mathrm{SU}(k), k \geq 2 & \mathrm{Ad}_{\mathrm{SU}(k)} & \\ \mathrm{SU}(k), k \geq 2 & a \mu_{k} \oplus b \mu_{k}^{*} & a+b=1 \\ & & a+b=2 \\ & & a+b=3,3 \mid k\end{array}$

$$
\begin{array}{lll}
\mathrm{S}_{\mathrm{p}}(3) & \Lambda^{2} \nu_{6} \\
\mathrm{~S}_{\mathrm{p}}(k), k \geq 3 & \nu_{2 k} & \\
& 3 \nu_{2 k} & \\
& { } \mathrm{~d}_{\mathrm{S}_{\mathrm{p}}(k)} & \\
\mathrm{S}_{\mathrm{PIN}}(5) & a \rho_{5} \oplus b \Delta_{5} & 2 a+b \mid 6, \quad b \neq 4 \\
\mathrm{~S}_{\mathrm{PIN}}(2 k+1), k \geq 2 & \rho_{2 k+1} & \\
& 3 \rho_{2 k+1} & \\
& A d_{\mathrm{S}_{\mathrm{PIN}}}(2 k+1) & a+b+c \mid 6, \quad b+c>0 \\
\mathrm{~S}_{\mathrm{PIN}}(8) & a \rho_{8} \oplus b \Delta_{8}^{+} \oplus c \Delta-1 \\
\mathrm{~S}_{\mathrm{PIN}}(10) & \Delta_{10}^{+} & \\
\mathrm{S}_{\mathrm{PIN}}(2 k), k \geq 4 & \rho_{2 k} & \\
& 2 \rho_{2 k} & \\
& 3 \rho_{2 k} & 3 \mid k-1 \\
& 6 \rho_{2 k} & \\
& A d_{\mathrm{S}_{\mathrm{PIN}}(2 k)}
\end{array}
$$




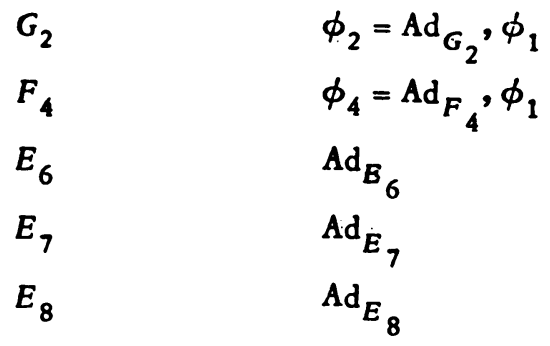

Proof. Let $\left\{\left(j, \mu_{j}\right): 1 \leq j \leq n\right\}=\Sigma(\lambda)$, and let $\sigma_{j}(\lambda)=\sigma_{j}\left(r_{\eta(T)}\left(\mu_{1}\right), \ldots, \tau_{\eta(T)}\left(\mu_{n}\right)\right)$ for $3 \leq j \leq 4$. Recall that

$$
\begin{aligned}
\left(\operatorname{ker} i^{*}\right)^{8}= & \left(Z v_{1} \oplus \cdots \oplus Z v_{l}\right)^{2} \sigma_{2}(\lambda) \oplus\left(Z v_{1} \oplus \cdots \oplus Z v_{l}\right) \sigma_{3}(\lambda) \\
& \oplus Z \sigma_{4}(\lambda) \text { where } l=\operatorname{rank} G
\end{aligned}
$$

Now

$$
P_{2}(\mathrm{SU}(n) / \mathrm{im} \lambda ; Z)+P_{1}(\mathrm{SU}(n) / \mathrm{im} \lambda ; Z) \cdot P_{1}\left(a_{\xi}\left(\operatorname{Ad}_{U}\right) ; Z\right)+P_{2}\left(a_{\xi}(\operatorname{Ad}) ; Z\right) \equiv \dot{0}
$$

(modulo 2 torsion). So as $P_{1}(\mathrm{SU}(n) / \mathrm{im} \lambda ; Z)=0=P_{2}(\mathrm{SU}(n) / \mathrm{im} \lambda ; Z)$ by hypothesis, we see that $\pi^{*}\left(P_{1}\left(a_{\xi}\left(\operatorname{Ad}_{U}\right) ; Z\right)\right)=0=\pi^{*}\left(P_{2}\left(a_{\xi}(\operatorname{Ad})_{U}\right) ; Z\right)$. Hence

$$
\left(\sum_{j=1}^{l} a_{j} v_{j}^{2}+\sum_{1 \leq i<j \leq l} b_{i j} v_{i} v_{j}\right) \sigma_{2}(\lambda)+\left(\sum_{j=1}^{l} d_{j} v_{j}\right) \sigma_{3}(\lambda)+m \cdot \sigma_{4}(\lambda)=\sigma_{4}\left(\mathrm{Ad}_{G}\right)
$$

where $a_{j}, b_{i j}, d_{j}, m \in Z$ for $1 \leq i \leq l, 1 \leq j \leq l$. Suppose $G=\mathrm{SU}(k)$. By using the two facts

$$
\sigma_{4}\left(y_{1},-y_{1}, \cdots, y_{p},-y_{p}\right)=\sigma_{2}\left(y_{1}^{2}, \ldots, y_{p}^{2}\right)
$$

and

$$
\sigma_{j}\left(X_{1}, \ldots, X_{p}, Y_{1}, \ldots, Y_{q}\right)=\sum_{a+\beta=j} \sigma_{a}\left(X_{1}, \ldots, X_{p}\right) \cdot \sigma_{\beta}\left(Y_{1}, \ldots, Y_{q}\right),
$$

it follows that

$$
\begin{aligned}
\sigma_{4}\left(\operatorname{Ad}_{\mathrm{SU}(k)}\right)=\left(2 k^{2}-k-6\right) & \left(\sigma_{1}\left(v_{1}^{4}, \ldots, v_{k-1}^{4}\right)+3 \sigma_{2}\left(v_{1}^{2}, \ldots, v_{k-1}^{2}\right)\right. \\
& \left.+2 \sum_{j=1}^{k-1} v_{j}^{2} \sigma_{2}\left(v_{1}, \ldots, \hat{v}_{j}, \ldots, v_{k-1}\right)\right) \\
& +\left(8 k^{2}-6 k-24\right) \sum_{j=1}^{k-1} v_{j} \sigma_{3}\left(v_{1}, \ldots, \hat{v}_{j}, \ldots, v_{k-1}\right) \\
& +\left(12 k^{2}-12 k-36\right) \sigma_{4}\left(v_{1}, \ldots, v_{k-1}\right)
\end{aligned}
$$

where $v_{j}=r_{\eta(T)}\left(\lambda_{j}\right)$ as usual. 
As an example of the method, consider $\lambda=a \mu_{k} \oplus b \mu_{k}^{*}, a+b \mid 2 k_{0}$ One shows easily that

$$
\begin{aligned}
& \sigma_{2}\left(\mu_{k}\right)=-\left(\sigma_{1}\left(v_{1}^{2}, \ldots, v_{k-1}^{2}\right)+\sigma_{2}\left(v_{1}, \ldots, v_{k-1}\right)\right), \\
& \sigma_{3}\left(\mu_{k}\right)=-\left(\sum_{j=1}^{k-1} v_{j}^{2} \sigma_{1}\left(v_{1}, \ldots, \hat{v}_{j}, \ldots, v_{k-1}\right)+2 \sigma_{3}\left(v_{1}, \ldots, v_{k-1}\right)\right), \\
& \sigma_{4}\left(\mu_{k}\right)=-\left(\sum_{j=1}^{k-1} v_{j}^{2} \sigma_{2}\left(v_{1}, \ldots, \hat{v}_{j}, \ldots, v_{k-1}\right)+3 \sigma_{4}\left(v_{1}, \ldots, v_{k-1}\right)\right), \\
& \sigma_{4}(\lambda)=(a+b) \sigma_{4}\left(\mu_{k}\right)+1 / 2(a+b)(a+b-1)\left(\sigma_{2}\left(\mu_{k}\right)\right)^{2} .
\end{aligned}
$$

So

$$
\begin{aligned}
\sigma_{4}\left(\mathrm{Ad}_{S U(k)}\right)= & -\left(\sum_{j=1}^{k-1} a_{j} v_{j}^{2}+\sum_{1 \leq i<j \leq k-1} b_{i j} v_{i} v_{j}\right) \\
& \cdot(a+b)\left(\sigma_{1}\left(v_{1}^{2}, \ldots, v_{k-1}^{2}\right)+\sigma_{2}\left(v_{1}, \ldots, v_{k-1}\right)\right) \\
& -(a-b) \sum_{j=1}^{k-1} d_{j} v_{j}\left(\sum_{j=1}^{k-1} v_{j}^{2} \sigma_{1}\left(v_{1}, \ldots, \hat{v}_{j}, \ldots, v_{k-1}\right)+2 \sigma_{3}\left(v_{1}, \ldots, v_{k-1}\right)\right) \\
& +m\left(\frac{1}{2}(a+b)(a+b-1) \sigma_{1}\left(v_{1}, \ldots, v_{k-1}\right)\right. \\
& +\frac{3}{2}(a+b)(a+b-1) \sigma_{2}\left(v_{1}^{2}, \ldots, v_{k-1}^{2}\right) \\
& +(a+b)(a+b-1) \sum_{j=1}^{k-1} v_{j}^{3} \sigma_{1}\left(v_{1}, \ldots, \hat{v}_{j}, \ldots, v_{k-1}\right) \\
& +(2(a+b)(a+b-1)-(a+b)) \sum_{j=1}^{k-1} v_{j}^{2} \sigma_{2}\left(v_{1}, \ldots, \hat{v}_{j}, \cdots, v_{k-1}\right) \\
& \left.+(3(a+b)(a+b-1)-3(a+b)) \sigma_{4}\left(v_{1}, \ldots, v_{k-1}\right)\right)
\end{aligned}
$$

Clearly, $a_{i}=a_{j}$ and $d_{i}=d_{j}$ if $1 \leq i, j \leq k-1$. Also, $b_{i j}=b_{a \beta}$ if $1 \leq i<j$ $\leq k-1,1 \leq a<\beta \leq k-1$. Note $-(a+b) a_{1}+1 / 2(a+b)(a+b-1) m=2 k^{2}-k-6$.

Suppose $k=2$. Then this is the only condition. So $-(a+b) a_{1}+1 / 2(a+b)$ $(a+b-1) m=0$. This is clearly acceptable for $a+b \mid 4$.

Suppose $k>2$.

$$
-(a+b)\left(a_{1}+a_{2}+b_{12}\right)-(a-b)\left(d_{1}+d_{2}\right)+3(a+b)(a+b-1) m / 2=6 k^{2}-3 k-18
$$

So $-(a+b) b_{12}-(a-b)\left(d_{1}+d_{2}\right)+1 / 2(a+b)(a+b-1) m=2 k^{2}-k=6$. Also, $-(a+b)\left(a_{1}+b_{12}\right)-(a-b) d_{1}+(a+b)(a+b-1) m=4 k^{2}-2 k-12$. So $-(a-b) d_{2}=0$. 
Hence $-(a+b) b_{12}+1 / 2(a+b)(a+b-1) m=2 k^{2}-k-6$.

Suppose $k=3$. Then this is the only condition. $-(a+b) a_{1}+1 / 2(a+b)(a+b-1) m=9$. So $a+b \mid 6$ is acceptable.

Suppose $k \geq 4 .-(a+b)\left(a_{1}+b_{12}+b_{13}+b_{23}\right)+(2(a+b)(a+b-1)-(a+b)) m=$ $8 k^{2}-6 k-24$ or $(a+b) m=2 k$. So

$$
\begin{aligned}
-(a+b) a_{1}+(a+b-1) k & =2 k^{2}-k-6 \\
-(a+b) a_{1} & =2 k^{2}-(a+b) k-6=2\left(k^{2}-3\right)-(a+b) k
\end{aligned}
$$

Hence $(a+b) \mid 2 k^{2}-6$. Recall $(a+b) \mid 2 k$. Hence $(a+b) \mid 2 k^{2}$. So $(a+b) \mid 6$. So $a \mu_{k} \oplus b \mu_{k}^{*}, k \geq 4$ is acceptable when $(a+b=1),(a+b=2),(a+b=3$ and $3 \mid k)$, $(a+b=6$ and $3 \mid k)$. This concludes the proof.

Remark. Suppose $\lambda: G_{1} \times G_{2} \rightarrow \operatorname{SU}(n)$ so that $P_{1}(\operatorname{SU}(n) /$ im $\lambda ; Z)=0$. Recall $\lambda=\Sigma_{j=1}^{t} \phi_{j} \otimes \psi_{j}$ where $\phi_{1}, \ldots, \phi_{t}, \psi_{1}, \ldots, \psi_{t}$ ar e either trivial or irreducible.

Let $T_{1}$ be a maximal torus for $G_{1}$, and $T_{2}$ for $G_{2}$. Let $v_{1}, \ldots, v_{\text {rk }} G_{1}$ be a base for $H^{2}\left(B_{T_{1}} ; Z\right)$, and $\bar{v}_{1}, \ldots, \bar{v}_{\mathrm{sk} G_{2}}$ for $H^{2}\left(B_{T_{2}} ; Z\right)$. For some nonzero integer $M$, we have

$$
\begin{aligned}
\sigma_{2}\left(\operatorname{Ad}_{G_{1}}\right)+\sigma_{2}\left(\operatorname{Ad} G_{2}\right) & =\sigma_{2}\left(\operatorname{Ad} G_{1} \times G_{2}\right)=M \cdot \sigma_{2}(\lambda)=M \cdot \sum_{j=1}^{t} \sigma_{2}\left(\phi_{j} \otimes \psi_{j}\right) \\
& =M \cdot \sum_{j=1}^{t}\left(\operatorname{dim} \psi_{j}\right) \sigma_{2}\left(\phi_{j}\right)+\left(\operatorname{dim} \phi_{j}\right) \sigma_{2}\left(\psi_{j}\right) \\
& =M \cdot \sum_{j=1}^{t}\left(\operatorname{dim} \psi_{j}\right) \sigma_{2}\left(\phi_{j}\right)+M \sum_{j=1}^{t}\left(\operatorname{dim} \phi_{j}\right) \sigma_{2}\left(\psi_{j}\right)
\end{aligned}
$$

So as $\sigma_{2}\left(\operatorname{Ad}_{G_{1}}\right)$ and $\sigma_{2}\left(\phi_{j}\right)(1 \leq j \leq t)$ are polynomials in $v_{1}, \ldots, v_{\text {sk } G_{1}}$, and $\sigma_{2}\left(\operatorname{Ad}_{G_{2}}\right)$ and $\sigma_{2}\left(\psi_{j}\right)(1 \leq j \leq t)$ are polynomials in $\bar{v}_{1}, \ldots, \bar{v}_{\text {rk } G_{2}}$, we have

$$
\begin{aligned}
& \sigma_{2}\left(\operatorname{Ad}_{G_{1}}\right)=M \sum_{j=1}^{t}\left(\operatorname{dim} \psi_{j}\right) \sigma_{2}\left(\phi_{j}\right), \text { and } \\
& \sigma_{2}\left(\operatorname{Ad}_{G_{2}}\right)=M \sum_{j=1}^{t}\left(\operatorname{dim} \phi_{j}\right) \sigma_{2}\left(\psi_{j}\right) .
\end{aligned}
$$

So as $n\left(\operatorname{Ad}_{G}\right)=0$ if and only if $G$ is a torus, we have the following.

Lemma 2.7. Let $H$ be a compact connected subgroup of $\mathrm{SU}(n)$ so that $P_{1}(\mathrm{SU}(n) / H)=0$. Then either $H$ is a torus or $H$ is semisimple.

So we may assume that $G_{1}$ and $G_{2}$ are simple. So if $P_{1}(\operatorname{SU}(n) /$ im $\lambda ; Z)=0=$ $P_{2}(\mathrm{SU}(n) /$ im $\lambda ; Z)$, then as before 


$$
\sigma_{4}\left(\operatorname{Ad}_{G_{1}}\right)+\sigma_{2}\left(\operatorname{Ad}_{G_{1}}\right) \sigma_{2}\left(\operatorname{Ad}_{G_{2}}\right)+\sigma_{4}\left(\operatorname{Ad}_{G_{2}}\right)=\sigma_{4}\left(\operatorname{Ad}_{G_{1} \times G_{2}}\right) \epsilon \operatorname{ker} i^{*} \cdot
$$

Theorem 2.9. Suppose that $\mathrm{SU}(n)$ acts as a differentiable transformation group on a connected manifold $M_{0}$ where $P_{1}(M ; Z)=0=P_{2}(M ; Z)$. Suppose also that $\operatorname{dim} M \leq n^{2}-8 n / 3-2$. Let $G$ be the connected component of the identity of "tbe" principal isotropy subgroup of the action. Then (possibly modulo a low-dimensional trivial representation), $G$ together with its embedding appears on the following list.

$\boldsymbol{G} \quad \lambda$

$$
\begin{aligned}
& \mathrm{SU}(k), \quad k \geq 4 \quad \mu_{k} \\
& k \geq 6 \quad a \mu_{k} \oplus b \mu_{k}^{*} \quad a+b=2 \\
& k \geq 9 \quad a \mu_{k} \oplus b \mu_{k}^{*} a+b=3,3 \mid k \\
& k \geq 18 a \mu_{k} \oplus b \mu_{k}^{*} a+b=6,3 \mid k \\
& \mathrm{SO}(2 k+1), k \geq 2 \quad \rho_{2 k+1} \\
& k \geq 113 \rho_{2 k+1} \quad 3 \mid 2 k-1 \\
& \mathrm{~S}_{\mathrm{P}}(k), \quad k \geq 3 \quad \gamma_{2 k} \\
& k \geq 8 \quad 3 \gamma_{2 k} \quad 3 \mid k+1 \\
& \mathrm{SO}(2 k), \quad k \geq 4 \quad \rho_{2 k} \\
& k \geq 6 \quad 2 \rho_{2 k} \\
& k \geq 10 \quad 3 \rho_{2 k} \quad 3 \mid k-1 \\
& k \geq 196 \rho_{2 k} \quad 3 \mid k-1
\end{aligned}
$$

Proof. Note that $n^{2}-8 n / 3-2 \geq \operatorname{dim} M \geq \operatorname{dim}(\operatorname{SU}(n) / \operatorname{im} \lambda)=\operatorname{dim} \operatorname{SU}(n)-\operatorname{dim} G$ $=n^{2}-1-\operatorname{dim} G$ and so $\operatorname{dim} G>8 n / 3$; that is, the dimension of $G$ as a Lie group is greater than $8 / 3$ times the dimension of $\lambda$ as a representation.

By Lemma 2.7, $G$ is either a torus or $G$ is semisimple. Suppose $G$ is a torus, say $G=T^{k}$. Then $k=\operatorname{dim} G>8 n / 3$. This is impossible as rk $\operatorname{SU}(n)=n-1$. So $G$ is semisimple.

We first suppose $G=G_{1} \times G_{2}$ where $G_{1}$ and $G_{2}$ are compact simple Lie groups. Recall $\lambda=\sum_{j=1}^{t} \phi_{j} \otimes \psi_{j}$. By 2.6 if for some $j$ boch $\phi_{j}$ and $\psi_{j}$ are nontrivial, then there exists a positive integer $M$ such that $n\left(\operatorname{Ad}_{G_{1}}\right) \geq M \cdot \operatorname{dim} \psi_{j} \cdot$ $n\left(\phi_{j}\right)$ and $n\left(\operatorname{Ad}_{G_{2}}\right) \geq M \cdot \operatorname{dim} \phi_{j} \cdot n\left(\psi_{j}\right)$. This turns out to be a severe restriction on $\phi_{j}$ and $\psi_{j}$. Using this fact together with 2.6 and 2.8 in the usual way, we arrive at the list for $G_{1} \times G_{2}$. We then use this list together with the additional 
requirement $\operatorname{dim} G>8 n / 3$ to prove that $G$ must be simple, and that, in fact, $(G, \lambda)$ lies on the above list.

3. The orbit types of $S U(n)$ actions on manifolds with vanishing first and second integral Pontrjagin classes.

Theorem 3.1. Let $\mathrm{SU}(n)$ act as a differentiable transformation group on a connected manifold $M$. Suppose that $P_{1}(M ; Z)=0=P_{2}(M ; Z)$, and that $\operatorname{dim} M \leq n^{2}-$ $8 n / 3$ - 2. Let $y \in M$ be a regular element and let $x \in M$. Then after conjugating $G_{y}^{0}$ if necessary, the inclusion $G_{y}^{0} \subseteq G_{x}^{0} \subseteq S U(n)$ appears on the following list:

(1) If

$$
\left(G_{y}^{0}\right)=\left(\mathrm{SU}(k) \stackrel{a \mu_{k} \oplus b \mu_{k}^{*} \oplus(n-(a+b)) \theta}{\longrightarrow} \mathrm{SU}(n)\right)
$$

where $a+b=1,2,3,6$ and $3 \mid k$ if $a+b=3,6$, then

$$
\left(G_{y}^{0} \subseteq G_{x}^{0} \subseteq \mathrm{SU}(n)\right)=\left(\mathrm{SU}(k) \stackrel{\mu_{k} \oplus(l-k) \theta}{\longrightarrow} \mathrm{SU}(l) \stackrel{a \mu_{l} \oplus b \mu_{l}^{*} \oplus(n-(a+b)) \theta}{\longrightarrow} \mathrm{SU}(n)\right) \text {. }
$$

(2) If

$$
\left(G_{y}^{0}\right)=\left(\mathrm{SO}(2 k+1) \stackrel{a \rho_{2 k+1} \oplus(n-(2 k+1) a) \Theta}{\longrightarrow} \mathrm{SU}(n)\right),
$$

where $a=1,3$ and $3 \mid(2 k-1)$ if $a=3$, then

$$
\begin{aligned}
& \left(G_{y}^{0} \subseteq G_{x}^{0} \subseteq \mathrm{SU}(n)\right)=\left(\mathrm{SO}(2 k+1) \stackrel{\rho_{2 k+1} \oplus(l-(2 k+1)) \theta}{\longrightarrow} \mathrm{SO}(2 l+1)\right. \\
& \left.\stackrel{a \rho_{2 k+1} \oplus(n-(a(2 l+1))) \theta}{\longrightarrow} \mathrm{SU}(n)\right)
\end{aligned}
$$

(3) If

$$
\left(G_{y}^{0}\right)=\left(S_{p}(k) \stackrel{a \gamma_{2 k} \oplus(n-2 a k) \theta}{\longrightarrow} S U(n)\right)
$$

where $a=1,3$ and $3 \mid(k-1)$ if $a=3$, then

$$
\left(G_{y}^{0} \subseteq G_{x}^{0} \subseteq S U(n)\right)=\left(\mathrm{S}_{\mathrm{p}}(k) \stackrel{\gamma_{2 k} \oplus 2(l-k) \theta}{\longrightarrow} \mathrm{S}_{\mathrm{p}}(l) \stackrel{a \gamma_{2 l} \oplus(n-2 a l) \theta}{\longrightarrow} \mathrm{SU}(n)\right) .
$$

(4) If

$$
\left(G_{y}^{0}\right)=\left(\mathrm{SO}(2 k) \stackrel{a \rho_{2 k} \oplus(n-2 a k) \theta}{\longrightarrow} \mathrm{SU}(n)\right)
$$

where $a=1,2,3,6$ and $3 \mid(k-1)$ if $a=3,6$, then

$$
\left(G_{y}^{0} \subseteq G_{x}^{0} \subseteq \operatorname{SU}(n)\right)=\left(\operatorname{SO}(2 k) \stackrel{\rho_{2 k} \oplus(n-2 k) \theta}{\longrightarrow} \mathrm{SO}(2 l) \stackrel{a \rho_{2 l} \oplus(n-2 a l) \theta}{\longrightarrow} \mathrm{SU}(n)\right) .
$$

Proof. As an example of the method, we shall give the proof for 


$$
\left(G_{y}^{0}\right)=\left(\mathrm{SU}(k) \stackrel{a \mu_{k} \oplus b \mu_{k}^{*} \oplus(n-2 k) \theta}{\longrightarrow} \mathrm{SU}(n)\right)
$$

where $a+b=2, a \geq 1$, and $k \geq 6$.

As $y$ is regular, after conjugating $G_{x}$ by a suitable element of $\operatorname{SU}(n)$, we may suppose $G_{y}^{0} \subseteq G_{x}^{0}$. Hence we first must study the lattice of closed connected subgroups $G_{y}^{0} \subseteq H \subseteq S U(n)$.

If $V$ is a subspace of $C^{n}$, let $S U(V)$ be the subgroup of $S U(n)$ which leaves $V^{\perp}$ fixed pointwise. Then in [5], the following theorem is proven.

Theorem. Let $V$ be a subspace of $\mathbf{C}^{n}$ with $\operatorname{dim} V \geq 3$. Let $H$ be a subgroup of $\mathrm{SU}(n)$ which contains $\mathrm{SU}(V)$. Then there exists a subspace $V_{1}$ of $\mathrm{C}^{n}$ with $V \subseteq V_{1}$ so that $\operatorname{SU}\left(V_{1}\right)$ is a normal subgroup of $H$.

We shall determine a similar result for $G_{y}^{0}$. Suppose $\phi: G_{y}^{0} \rightarrow H, \psi: H \rightarrow$ $\mathrm{SU}(n)$, and $\lambda=\phi \circ \psi$. We may take $H=T^{c} \times U_{1} \times \cdots \times U_{d}$ where $T^{c}$ is a torus of rank $c, U_{j}$ is a compact, simply connected, simple Lie group, and where $\phi$ and $\psi$ have finite kernels. Then $\phi=\phi_{T} \oplus \phi_{1} \oplus \cdots \oplus \phi_{d^{\circ}}$ Clearly $\phi_{T}$ is trivial as $\mathrm{SU}(k)$ is simple and $T^{c}$ is abelian. So we may take $V=U_{1} \times \cdots \times U_{d}$, and $\phi=$ $\phi_{1} \oplus \cdots \oplus \phi_{d}$. Now $\psi=\Sigma_{j=1}^{t} \psi_{j_{1}} \otimes \cdots \otimes \psi_{j_{d}}$ where each $\psi_{j_{i}}$ is either trivial or irreducible.

Let $(\psi \circ \phi)_{j}=\left(\psi_{j_{1}} \circ \phi_{1}\right) \otimes \cdots \otimes\left(\psi_{j_{d}} \circ \phi_{d}\right)$. Note that $(\psi \circ \phi)_{j}$ is a nontrivial representation for some $j$.

For some $1 \leq i \leq m, \psi_{j_{i}} \circ \phi_{i}: \mathrm{SU}(k) \rightarrow \mathrm{SU}(n)$ is nontrivial. Note that the nontrivial components of $\psi_{j_{i}} \circ \phi_{i}$ have dimension $\geq k_{0}$. Also if there exists $1 \leq a \leq m$ with $i \neq \alpha$ and $\psi_{j_{a}} \circ \phi_{a}$ nontrivial, then the nontrivial components of $(\psi \circ \phi)_{j}$ have dimension $\geq k^{2}$ which is greater than $2 k$.

So as $\psi \circ \phi=a \mu_{k} \oplus b \mu_{k}^{*} \oplus(n-2 k) \theta$ and $a+b=2$, it is clear that we may assume

(1) $(\psi \circ \phi)_{j}$ is trivial if $j>2$.

(2) If $j=1,2$, then there exists at most one integer $i_{j}$ with $1 \leq i_{j} \leq m$ so that $\psi_{j_{i j}} \circ \phi_{i_{j}}$ is nontrivial.

So $G_{y}^{0}$ is contained in a normal subgroup $V$ of $H$, where $V=U$, or $V=$ $U_{1} \times U_{2}$

Suppose $V=\operatorname{SU}(l)$. Then we may write $\phi: \operatorname{SU}(k) \rightarrow \operatorname{SU}(l), \psi: \operatorname{SU}(l) \rightarrow \operatorname{SU}(n)$, and $\psi \circ \phi=\lambda=a \mu_{k} \oplus b \mu_{k}^{*} \oplus(n-2 k) \theta$, where $a+b=2$. So if we write $\psi=\psi_{1} \oplus$ $\cdots \oplus \psi_{t} \oplus L \theta: \mathrm{SU}(l) \rightarrow \mathrm{SU}(n)$, where $\psi_{j_{*}}$ is irreducible for $1 \leq j \leq t$, then $\left(\psi_{1} \circ \phi\right)$ $\oplus \cdots \oplus\left(\psi_{t} \circ \phi\right) \oplus L \theta=\psi \circ \phi=a \mu_{k} \oplus b \mu_{k}^{*} \oplus(n-2 k) \theta$.

Hence $\psi=\psi_{1} \oplus L \theta$ or $\psi=\psi_{1} \oplus \psi_{2} \oplus L \theta$.

Now $l^{2}-1 \geq k^{2}-1>8 n / 3$. Hence $\psi_{1}$ and $\psi_{2}$ have drastic limitations on their dimensions. Using the fact that $\phi>\psi$ implies $\operatorname{dim} \phi>\operatorname{dim} \psi$, it follows 
that the only possible irreducible components of $\psi$ are $\mu_{l}$ and $\mu_{l}^{*}$ Arguing similarly for $\phi$, we see that the only possible irreducible components of $\phi$ are $\mu_{k}$ and $\mu_{k}^{*}$.

Hence, it is clear that one of the following cases holds:

(1) $\phi=a \mu_{k} \oplus b \mu_{k}^{*} \oplus(l-2 k) \theta, \psi=\mu_{l} \oplus(n-l) \theta$.

(2) $\phi=b \mu_{k} \oplus a \mu_{k}^{*} \oplus(l-2 k), \psi=\mu_{l}^{*} \oplus(n-l) \theta$.

(3) $\phi=\mu_{k} \oplus(l-k) \theta, \psi=a \mu_{l} \oplus b \mu_{l}^{*} \oplus(n-2 l) \theta$.

(4) $\phi=\mu_{k}^{*} \oplus(l-k) \theta, \psi=b \mu_{l} \oplus a \mu_{l}^{*} \oplus(n-2 l) \theta$.

Arguing similarly for the case when $V$ is some other simple compact Lie group (and using the fact that $\operatorname{dim} V>k^{2}-1>8 n / 3$ ), we get the following additional possibilities, where $\phi: \mathrm{SU}(k) \rightarrow V, \psi: V \rightarrow \mathrm{SU}(n)$, and $\psi \circ \phi=\lambda$.

V

$S_{p}(l)(a=1=b)$

$\operatorname{SO}(l)(a=1=b)$ $\phi$

$$
\mu_{k} \oplus \mu_{k}^{*} \oplus 2(l-k) \theta
$$$$
\mu_{k} \oplus \mu_{k}^{*} \oplus(l-2 k) \theta
$$

$\psi$

$\gamma_{2 l} \oplus(n-2 l) \theta$

$\rho_{l} \oplus(n-l) \theta$

Now suppose $V=U_{1} \times U_{2}$. Then using arguments similar to the above, one shows that $V=\operatorname{SU}\left(l_{1}\right) \times \operatorname{SU}\left(l_{2}\right)$, and that we can write $\phi=\phi_{1} \oplus \phi_{2}$ and $\psi=$ $\left(\psi_{1} \otimes \theta\right) \oplus\left(\theta \otimes \psi_{2}\right)$ where modulo trivial copies,

$$
\begin{array}{ll}
\phi_{1}=\mu_{k}, \mu_{k}^{*}, & \phi_{2}=\mu_{k}, \mu_{k}^{*}, \\
\psi_{1}=\mu_{l_{1}}, \mu_{l_{1}}^{*}, & \psi_{2}=\mu_{l_{2}}, \mu_{l_{2}}^{*} .
\end{array}
$$

We now specialize to the case $H=G_{x}^{0}$.

We shall show that $H=G_{x}^{0}$ implies ${ }^{V}=\mathrm{SU}(l)$, and that in fact

$$
\left(G_{y}^{0} \subset V \subset \mathrm{SU}(n)\right)=\left(\mathrm{SU}(k) \stackrel{\mu_{k} \oplus(l-k) \theta}{\longrightarrow} \mathrm{SU}(l) \stackrel{a \mu_{l} \oplus b \mu_{l}^{*} \oplus(n-2 k) \theta}{\longrightarrow} \mathrm{SU}(n)\right) .
$$

Let $\xi_{1}=\left(G_{x} \rightarrow G \rightarrow G / G_{x}\right)$. Then for $j=1,2$,

$$
0=P_{j}\left(r\left(G / G_{x}\right) \oplus \nu\left(G / G_{x}\right)\right)=P_{j}\left(\alpha_{\xi}\left(A_{1} \mid G_{x}-A_{d_{x}}\right) \oplus \alpha_{\xi_{1}}\left(\phi_{x}\right)\right)
$$

Now $V$ is a connected normal subgroup of $G_{x}^{0}$ so that

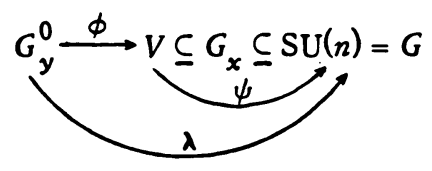

i.e. $\psi \circ \phi=\lambda$.

Let $\pi: G / V \rightarrow G / G_{x}$ be the natural map.

Let $\xi_{2}=(V \stackrel{\psi}{\longrightarrow} G \rightarrow G / V)$. Then if $\Gamma$ is a real representation of $G_{x}$, 


$$
\begin{aligned}
& \pi^{!}\left(\alpha_{\xi_{1}}(\Gamma)\right)=\alpha_{\xi_{2}}(\Gamma \mid V) \text {. So } \\
& \pi^{!}\left(a_{\xi_{1}}\left(\operatorname{Ad} G \mid G_{x}-\operatorname{Ad}_{G_{x}}\right) \oplus a_{\xi_{1}}\left(\phi_{x}\right)\right)=a_{\xi_{2}}\left(\operatorname{Ad}{ }_{G}\left|V-\operatorname{Ad}_{G_{x}}\right| V\right) \oplus a_{\xi_{2}}\left(\phi_{x} \mid V\right) \\
& =a_{\xi_{2}}\left(\operatorname{Ad}_{G} \mid V-\operatorname{Ad} V-\theta\right) \oplus a_{\xi_{2}}\left(\phi_{x} \mid V\right) \\
& =r(G / V) \oplus a_{\xi_{2}}\left(\phi_{x} \mid V\right) \in \tilde{K O}(G / V) \text {, }
\end{aligned}
$$

where $\theta$ is some trivial representation of $V$. So modulo torsion,

$$
P_{1}(G / V)+P_{1}\left(\alpha_{\xi_{2}}\left(\phi_{x} \mid V\right)\right)=0,
$$

and

$$
P_{2}\left(G^{\prime} V\right)+P_{1}\left(G^{\prime} V\right) \cdot P_{1}\left(a_{\xi_{2}}\left(\phi_{x} \mid V\right)\right)+P_{2}\left(a_{\xi_{2}}\left(\phi_{x} \mid V\right)\right)=0 \text {. }
$$

Recall that as $G_{y}$ is the principal isotropy subgroup of $\phi_{x}, \phi_{x}\left|G_{y}=A d_{G_{x}}\right| G_{y}-$ $\operatorname{Ad}_{G_{y}} \oplus$ trivial copies. So

$$
\begin{aligned}
\phi_{x} \mid G_{y}^{0} & =\operatorname{Ad}_{G_{x}}\left|G_{y}^{0}-\mathrm{Ad}_{G_{y}}\right| G_{y}^{0} \oplus \text { trivial copies } \\
& =\left(\left(\operatorname{Ad}_{G_{x}} \mid G_{x}^{0}\right) \mid V\right) \mid G_{y}^{0}-\mathrm{Ad}_{G_{y}^{0}} \oplus \text { trivial copies } \\
& =\operatorname{Ad}_{V} \mid G_{y}^{0}-\mathrm{Ad}_{G_{y}^{0}} \oplus \text { trivial copies } \\
& =\operatorname{Ad}_{V} \circ \phi-\mathrm{Ad}_{G_{y}^{0}} \oplus \text { trivial copies. }
\end{aligned}
$$

Note also that $\operatorname{dim}\left(\iota_{\mathrm{SU}(n) / G_{x}} \oplus \phi_{x}\right)=\operatorname{dim} M \leq n^{2}-8 n / 3-2$, and hence $\operatorname{dim} \phi_{x} \leq$ $n^{2}-8 n / 3-2$.

We shall now examine the several possibilities for $V, \phi$, and $\psi$.

Suppose $V=\mathrm{SU}(l)$, and $\phi=a \mu_{k} \oplus b \mu_{k}^{*} \oplus(l-2 k) \theta, \psi=\mu_{l} \oplus(n-l) \theta$. Then

$$
\begin{aligned}
\phi_{x} \mid \mathrm{SU}(k)= & \phi_{x} \circ \phi=\left(\mu_{l} \otimes \mu_{l}^{*}-\theta\right) \circ \phi-\mu_{k} \otimes \mu_{k}^{*} \oplus \text { trivial copies } \\
= & a b\left(\mu_{k} \otimes \mu_{k} \oplus \mu_{k}^{*} \otimes \mu_{k}^{*}\right) \oplus\left(a^{2}+b^{2}-1\right) \mu_{k} \otimes \mu_{k}^{*} \\
& \oplus(a+b)(l-(a+b) k)\left(\mu_{k} \oplus \mu_{k}^{*}\right) \oplus \text { trivial copies. }
\end{aligned}
$$

Recall $k^{2}-1>8 n / 3$, and $l \geq 2 k$. So $\operatorname{dim} \phi_{x}<n^{2}<9 k^{4} / 64<9 l^{4} / 1024$.

This is a severe limitation on $\phi_{x}$. By listing all the irreducible representations $\Gamma$ of $\operatorname{SU}(l)$ with $\operatorname{dim} \Gamma<9 l^{4} / 1024$ and then using our determination of $\phi_{x} \circ \phi$, we see easily that $\phi_{x} \mid \mathrm{SU}(l)$ can contain copies only of $\mu_{l}, \mu_{l}^{*}, \mu_{l} \otimes \mu_{l}$, $\mu_{l} \otimes \mu_{l}^{*}, \mu_{l}^{*} \otimes \mu_{l^{\circ}}^{*}$ Hence 


$$
\phi_{x} \mid \mathrm{SU}(l)=c\left(\mu_{l} \otimes \mu_{l} \oplus \mu_{l}^{*} \otimes \mu_{l}^{*}\right) \oplus d \mu_{l} \otimes \mu_{l}^{*} \oplus e\left(\mu_{l} \oplus \mu_{l}^{*}\right) \oplus F \theta \text {, }
$$

and so $\phi_{x} \mid \operatorname{SU}(k)=\phi_{x} \circ \phi=\left(4 a b c+\left(a^{2}+b^{2}\right) d\right) \mu_{k} \otimes \mu_{k}^{*} \oplus$ a sum of representations not containing $\mu_{k} \otimes \mu_{k}^{*}$. So $4 a b c+\left(a^{2}+b^{2}\right) d=a^{2}+b^{2}-1$. This is impossible.

In the other cases for $V, \phi, \psi$, we argue similarly to the above. In some cases there will exist a representation $\phi_{x} \mid V$ so that $\phi_{x} \circ \phi=\lambda$. In these cases, by using $P_{1}(G / V)+P_{1}\left(a_{\xi_{2}}\left(\phi_{x} \mid V\right)\right)=0$, we arrive at the conclusion that $V=S U(l)$, $\phi=\mu_{k} \oplus(l-k) \theta$, and $\psi^{2}=a \mu_{l} \oplus b \mu_{l}^{*}$, i.e.

$$
\left(G_{y}^{0} \subseteq V \subseteq S U(n)\right)=\left(\operatorname{SU}(k) \stackrel{\mu_{k} \oplus(l-k) \theta}{\longrightarrow} \mathrm{SU}(l) \stackrel{a \mu_{l} \oplus b \mu_{l}^{*} \oplus(n-2 k) \theta}{\longrightarrow} \mathrm{SU}(n)\right)
$$

as was to be shown.

Finally we shall show $G_{x}^{0}=V$.

So suppose $G_{x}^{0} \neq V$. We shall derive a contradiction. We give here a modification of an argument found in [4].

We may suppose $x$ was chosen so that $\operatorname{dim} G_{x}$ is minimal among these $G_{t}$ with $G_{t}^{0}$ not simple. We see that after conjugating if necessary, we have $y \in S_{x}$, the slice at $x$. Let $\phi_{x}$ be the slice representation of $G_{x}$ at $x_{0}$. Then $\phi_{x} \mid G_{x}^{0}=\phi_{1} \oplus \beta \theta$, where $\phi_{1}$ is a nontrivial representation without trivial copies.

We may write $y=\bar{y}+n$ where $\bar{y} \in W_{\phi_{1}}$, the representation space of $\phi_{1}$, and where $\eta \in\left(W_{\phi_{1}}\right)^{\perp}$ i.e. $\eta \in W_{\beta \theta^{\circ}}$

Clearly $G_{y}=G_{\bar{y}}$ and so $\bar{y}$ is a regular element.

Let $z$ be an element of the unit ball of $w_{\phi_{1}} \subseteq S_{x} \subseteq M_{\text {. To show: }}\left(G_{x}^{0}\left(\phi_{1}\right)_{z}\right)^{0}$ $\subseteq V$. By the minimality condition

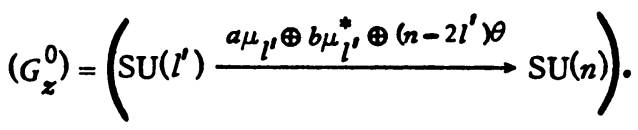

So as $G_{z}^{0} \subseteq G_{x}^{0} \subseteq N_{\text {SU(n) }}(V)$, clearly $G_{z}^{0} \subseteq V$. Hence $\left(G_{x}^{0}\left(\phi_{1}\right)_{z}\right)^{0}=G_{z}^{0} \subseteq V$. So we may apply the following proposition found in [4].

Proposition. Let $H$ be a closed, connected, proper normal subgroup of a compact, connected, Lie group $K$. C.H. Then

Let $\psi: K \rightarrow O(m)$ be a representation, such that for each $x \in W_{\psi}-\{0\}, K_{x}^{0}$

(1) $H \subseteq$ ker $\psi$.

(2) $\operatorname{rank} K / H=1$. 
Hence $V \subseteq \operatorname{ker} \phi_{1}=\operatorname{ker} \phi_{x} \mid G_{x}^{0}$ and rank $G_{x}^{0} / V=1$. So $\phi_{x} \mid G_{y}^{0}$ is trivial. But note that $\phi_{x} \circ \phi=(l-k)\left(\mu_{k} \oplus \mu_{k}^{*}\right) \oplus$ trivial copies, as

$$
\begin{aligned}
\phi_{x} \mid G_{y}^{0} & =\left(\phi_{x} \mid G_{y}\right)\left|G_{y}^{0}=\left(\Lambda d_{G_{x}} \mid G_{y}-\Lambda d_{G_{y}}\right)\right| G_{y}^{0} \\
& =\left(\Lambda d_{G_{x}} \mid V\right)\left|G_{y}^{0}-\Lambda d_{G_{y}}\right| G_{y}^{0} \\
& =\Lambda d_{V} \mid G_{y}^{0}-\Lambda \mathrm{d}_{G_{y}^{0}} \oplus \theta .
\end{aligned}
$$

Hence $l=k$, and so $V=G_{y}^{0}$. So $G_{x}^{0} \sim G_{y}^{0} \times L$, where either $L=\operatorname{SU}(2)$ or $L=T^{1}$.

Note that $\phi_{x} \mid G_{x}^{0}=\theta \otimes y_{0}$. Let $\left\{a_{1}\right\}$ be the standard base for "the" Cartan subalgebra of $L$. Suppose

$$
\left(G_{x}^{0} \rightarrow \mathrm{SU}(n)\right)=\left(S U(k) \times L \stackrel{\left.\left(a \mu_{k} \oplus b \mu_{k}^{*} \oplus t \theta\right\} \oplus \Gamma\right) \otimes(\theta \otimes \delta)}{\longrightarrow} \mathrm{SU}(n)\right) .
$$

Then $\operatorname{dim} \Gamma=1$. So $\Gamma=\theta \propto L=T^{1}$. Suppose $\Gamma=\theta$. Let $\xi_{3}=\left(G_{x}^{0} \rightarrow \operatorname{SU}(n) \rightarrow \operatorname{SU}(n) / G_{x}^{0}\right)$ Then

$$
P_{1}\left(a_{\xi_{3}}\left(l \operatorname{su(n)/G_{x}^{0}}\right) \oplus a_{\xi_{3}}\left(\phi_{x} \mid G_{x}^{0}\right)\right)=0
$$

So

$$
\begin{aligned}
& M\left(2 \sigma_{2}\left(\mu_{k}\right)+\sigma_{2}(\delta)\right)=\sigma_{2}\left(l_{\mathrm{SU}(n) / G_{x}^{0}}\right)+\sigma_{2}\left(\phi_{x} \mid G_{x}^{0}\right) \\
& =\sigma_{2}\left(\left(\left(\left(a \mu_{k} \oplus b \mu_{k}^{*} \oplus t \theta\right) \otimes \theta\right) \oplus(\theta \otimes \delta)\right)\right. \\
& \left.\quad \otimes\left(\left(\left(a \mu_{k}^{*} \oplus b \mu_{k} \oplus t \theta\right) \otimes \theta\right) \oplus\left(\theta \otimes \delta^{*}\right)\right)-\mu_{k}^{\prime} \otimes \mu_{k}^{*}-\Lambda d_{L}\right)+\sigma_{2}(\gamma) \\
& =(6 k+4 t+4 \operatorname{dim} \delta) \sigma_{2}\left(\mu_{k}\right)+(4 k+2 t) \sigma_{2}(\delta)+2 \operatorname{dim} \delta \cdot \sigma_{2}(\delta)-\sigma_{2}\left(\Lambda d_{L}\right)+\sigma_{2}(y) .
\end{aligned}
$$

Hence $2 M=6 k+4 t+4 \operatorname{dim} \delta$. So

$$
(3 k+2 t+2 \operatorname{dim} \delta) \sigma_{2}(\delta)=(4 k+2 t) \sigma_{2}(\delta)+2 \operatorname{dim} \delta \cdot \sigma_{2}(\delta)-\sigma_{2}\left(\operatorname{Ad}_{L}\right)+\sigma_{2}(\gamma)
$$

or

$$
-k \sigma_{2}(\delta)=\sigma_{2}(\gamma)-\sigma_{2}\left(\mathrm{Ad}_{L}\right)
$$

So as $\sigma_{2}(\delta), \sigma_{2}(\gamma), \sigma_{2}\left(\Lambda d_{L}\right)$ are all polynomials in $r\left(a_{1}\right)$ with negative coefficients, we see $L=\mathrm{SU}(2)$. So $\sigma_{2}\left(\Lambda \mathrm{d}_{\mathrm{SU}(2)}\right)=\sigma_{2}(y)+k \sigma_{2}(\delta)$. An easy check of $R O(S U(2))$ shows this is impossible.

So $\Gamma \neq \theta$. Hence $L=T^{1}$. By considering $L \hookrightarrow \operatorname{SU}(n)$, we see $(2 k+t) \Gamma \oplus \delta$ : 
$L \rightarrow \mathrm{SU}(n)$. We easily have

$$
\begin{aligned}
& M\left(2 \sigma_{2}\left(\mu_{k}\right)+\sigma_{2}((2 k+t) \Gamma \oplus \delta)\right)=M \sigma_{2}\left(\left(\left(a \mu_{k} \oplus b \mu_{k}^{*} \oplus t \theta\right) \otimes \Gamma\right) \oplus(\theta \otimes \delta)\right) \\
& =\sigma_{2}\left(\left(\left(\left(a \mu_{k} \oplus b \mu_{k}^{*} \oplus t \theta\right) \otimes \Gamma\right) \oplus(\theta \otimes \delta)\right) \otimes\left(\left(\left(a \mu_{k}^{*} \oplus b \mu_{k} \oplus t \theta\right) \otimes \Gamma^{*}\right) \oplus\left(\theta \otimes \delta^{*}\right)\right)-\mu_{k} \otimes \mu_{k}^{*}\right)+\sigma_{2}(\gamma) \\
& =\sigma_{2}\left(\left(a^{2}+b^{2}-1\right) \mu_{k} \otimes \mu_{k}^{*} \oplus a b\left(\mu_{k} \otimes \mu_{k} \oplus \mu_{k}^{*} \otimes \mu_{k}^{*}\right) \oplus(a+b) t\left(\mu_{k} \oplus \mu_{k}^{*}\right)\right. \\
& \quad \quad+\sigma_{2}\left((2 k+t)\left(\Gamma \otimes \mu_{k} \oplus b \mu_{k}^{*} \oplus t \theta\right) \otimes\left(\Gamma \otimes \delta^{*}\right) \oplus\left(a \mu_{k}^{*} \oplus b \mu_{k} \oplus t \theta\right) \oplus\left(\Gamma^{*} \otimes \delta\right) \oplus \theta \otimes\left(\delta \otimes \delta^{*}\right)\right)+\sigma_{2}(\gamma) \\
& =\left(2 k\left((a+b)^{2}-1\right)+2(a+b) t+2(a+b) \operatorname{dim} \delta\right) \sigma_{2}\left(\mu_{k}\right) \\
& =(6 k+4 t+4 \operatorname{dim} \delta) \sigma_{2}\left(\mu_{k}\right)+\sigma_{2}\left(((2 k+t) \Gamma \oplus \delta) \otimes\left((2 k+t) \Gamma^{*} \oplus \delta^{*}\right)\right) \\
& =(6 k+4 t+4 \operatorname{dim} \delta) \sigma_{2}\left(\mu_{k}\right)+(4 k+2 t+2 \operatorname{dim} \delta) \sigma_{2}((2 k+t) \Gamma \oplus \delta)+\sigma_{2}(\gamma) .
\end{aligned}
$$

So $2 M=6 k+4 t+4 \operatorname{dim} \delta$, or $M=3 k+2 t+2 \operatorname{dim} \delta$. Hence

$$
(3 k+2 t+2 \operatorname{dim} \delta) \sigma_{2}((2 k+t) \Gamma \oplus \delta)=(4 k+2 t+2 \operatorname{dim} \delta) \sigma_{2}((2 k+t) \Gamma \oplus \delta)+\sigma_{2}(\gamma)
$$

So $0=k \sigma_{2}((2 k+t) \Gamma \oplus \delta)+\sigma_{2}(\gamma)$. But as $\phi_{x} \mid G_{x}^{0}=\theta \otimes \gamma$, we see that $\gamma \otimes \mathrm{C}$ : $L \rightarrow \operatorname{SU}(n)$, and hence $k \sigma_{2}((2 k+t) \Gamma \oplus \delta)$ and $\sigma_{2}(\gamma)$ are polynomials in $\tau\left(a_{1}\right)$ with negative coefficients. This is impossible.

The other cases follow similarly.

\section{REFERENCES}

1. A. Borel et al., Seminar on transformation groups, Ann. of Math. Studies, no. 46, Princeton Univ. Press, Princeton, N. J., 1961.

2. A. Borel and F. Hirzebruch, Characteristic classes and homogeneous spaces. I, Amer. J. Math. 80 (1958), 458-538; II, 81 (1959), 351-382; III, 82 (1960), 491-504. MR 21 \#1586; 22 \#988; 22 \#11413.

3. E. A. Grove, $\mathrm{SU}(n)$ actions on manifolds with vanishing first and second integral pontrjagin classes, Proc. Second Conference on Transformation Groups, Amherst, 1971, Springer, 1972, pp. 324-333.

4. W. C. Hsiang and W. Y. Hsiang, Differentiable actions of compact connected classical groups. I, Amer. J. Math. 89 (1967), 705-786; II, Ann. of Math. (2) 92 (1970), 189223. MR 36 \#304; 42 \#420.

5. W. Y. Hsiang, On the principal orbit type and P. A. Smith theory of $\mathrm{SU}(p)$ actions, Topology 6 (1967), 125-135. MR 34 \#5084.

6. R. S. Palais, The classification of G-spaces, Mem. Amer. Math. Soc. No. 36 (1960). MR $31 \# 1664$.

DEPARTMENT OF MATHEMATICS, UNIVERSITY OF RHODE ISLAND, KINGSTON, RHODE ISLAND 02881 